\title{
Effects of substituents and molecular weight on the optical, thermal and photovoltaic properties of alternating dithienogermole-dithienylbenzothiadiazole polymers
}

\author{
Fei-Bao Zhang ${ }^{1}$, Joji Ohshita ${ }^{1}$, Masayuki Miyazaki ${ }^{1}$, Daiki Tanaka ${ }^{1}$ and Yasushi Morihara ${ }^{2}$ \\ Polymer Journal (2014) 46, 628-631; doi:10.1038/pj.2014.35; published online 28 May 2014
}

Keywords: bulk heterojunction polymer solar cell; dithienogermole; donor-acceptor interaction; $\pi$-conjugated polymer

\begin{abstract}
INTRODUCTION
Bulk heterojunction-type polymer solar cells (BHJ-PSCs) are of current interest because of their potential applications in lightweight and flexible modules. Simple fabrication by a solution process is also an advantage of BHJ-PSC. In this system, a film consisting of a blend of an electron-donating host polymer and an electron-accepting guest material, such as 6,6-phenyl- $\mathrm{C}_{61}$-butyric acid methyl ester $\left(\mathrm{PC}_{61} \mathrm{BM}\right)$ and its $\mathrm{C}_{70}$ analogue $\mathrm{PC}_{71} \mathrm{BM}$, is employed as the active layer, where donor-acceptor (D-A)-type-conjugated polymers have been extensively studied as the host polymers. ${ }^{1}$ The broad and red-shifted absorptions of $\mathrm{D}-\mathrm{A}$ type polymers make it possible to utilize the wide range of wavelengths in sunlight. However, the photovoltaic properties of the polymer are dependent not only on its structure but also on its molecular weight..$^{2-5}$ It is commonly accepted that higher polymer molecular weight leads to better carrier-transporting properties, which relates to the cell current density, as long as the polymer exhibits sufficiently high solubility for the solution process. To overcome the solubility problem arising from increased molecular weight, solubilizing alkyl or alkoxy pendant groups are introduced onto the polymer chain. However, the introduction of these pendant groups often changes the electronic structure of the polymer and the film morphology. Thus, fine-tuning of the substitution mode is required to improve the performance. ${ }^{6}$

Dithienosilole (DTS) has been studied as a promising donor component of D-A type polymers for $\mathrm{BHJ}-\mathrm{PSCs}^{7}$ because of its interesting electronic state. ${ }^{8}$ Yang and colleagues ${ }^{7,9-16}$ prepared a DTSbenzothiadiazole (BT) alternating copolymer (pDTS-BT in Scheme 1) and demonstrated its efficient photovoltaic properties. Its highly planar structure results in efficient $\pi$ conjugation. In addition, an inphase interaction between the silicon $\sigma^{*}$ - and the bithiophene $\pi^{*}$ orbitals stabilizes the LUMO (lowest unoccupied molecular orbital),
\end{abstract}

whereas the HOMO (highest occupied molecular orbital) remains nearly intact to further minimize the HOMO-LUMO energy gap. This is a suitable electronic state (that is, a low band gap and lowlying HOMO) for photovoltaic host polymers, as it leads to wide range absorption and high voltage in the cells. Recently, we introduced dithienogermole (DTG) as a new donor component for D-A type polymers, hypothesizing that the introduction of the heavier germanium atom in place of silicon would provide stronger chain-chain interactions and enhance the carrier mobility in the polymer films. ${ }^{17-19}$ At nearly the same time, Reynolds and colleagues. ${ }^{20,21}$ also reported the synthesis of DTG-containing photovoltaic polymers. ${ }^{20,21}$ As expected, some DTG-containing polymers exhibited superior photovoltaic performance in the BHJPSC system compared with DTS analogs, primarily because of their higher current density. ${ }^{18,20}$ In the course of our studies to further improve the photovoltaic properties of DTG-containing polymers, we demonstrated that the performance of BHJ-PSCs based on a DTG-BT alternating copolymer (pDTG-BT) was significantly improved by increasing the polymer molecular weight (Scheme 1). ${ }^{17}$ It was also demonstrated that an alternating polymer with DTG and dithienylbenzothiadiazole (TBT) units (pDTG-TBT) exhibited better photovoltaic properties than pDTG-BT, as shown in Scheme $1 .{ }^{19}$ However, the molecular weight of the previously prepared pDTGTBT was only $M_{\mathrm{n}}=3800$.

Here, we report the synthesis of new DTG-TBT polymers with solubilizing alkyl substituents on the 2,5-thienylene units in TBT. These polymers showed very high molecular weights of $M_{n}=26000$ 57000 . Reinvestigation of the synthesis of pDTG-TBT to increase the molecular weight by using a monomer purified by gel permeation chromatography (GPC) was also examined. BHJ-PSCs were fabricated using the present polymers and their performance was evaluated to

${ }^{1}$ Department of Applied Chemistry, Graduate School of Engineering, Hiroshima University, Higashi-Hiroshima, Japan and ${ }^{2}$ Synthesis Research Laboratory, Kurashiki Research Center, Kuraray Co, Ltd, Kurashiki, Japan

Correspondence: Professor J Ohshita, Department of Applied Chemistry, Graduate School of Engineering, Hiroshima University, Higashi-Hiroshima 739-8527, Japan. E-mail: jo@hiroshima-u.ac.jp

Received 6 January 2014; revised 25 March 2014; accepted 26 March 2014; published online 28 May 2014 


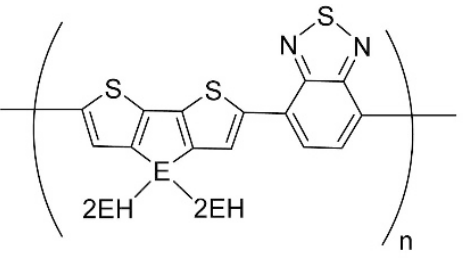

pDTS-BT E $=\mathrm{Si}$

pDTG-BT $E=\mathrm{Ge}$

$M_{\mathrm{n}}=8,000, M_{\mathrm{w}} / M_{\mathrm{n}}=1.38, \mathrm{PCE}=0.71 \%$

$M_{\mathrm{n}}=15,000, M_{\mathrm{w}} / M_{\mathrm{n}}=1.67, \mathrm{PCE}=1.21 \%$

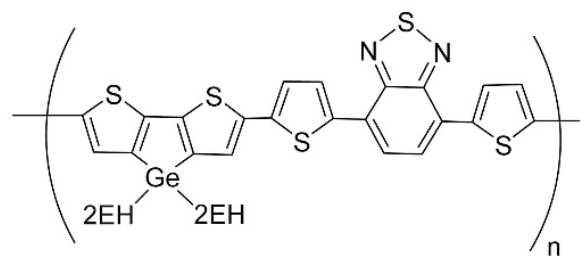

pDTG-TBT

$M_{\mathrm{n}}=3,800, M_{\mathrm{w}} / M_{\mathrm{n}}=3.15, \mathrm{PCE}=1.55 \%$

Table 1 Preparation of DTG-TBT polymers

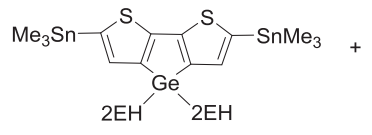

DTGn

2EH = 2-ethylhexy

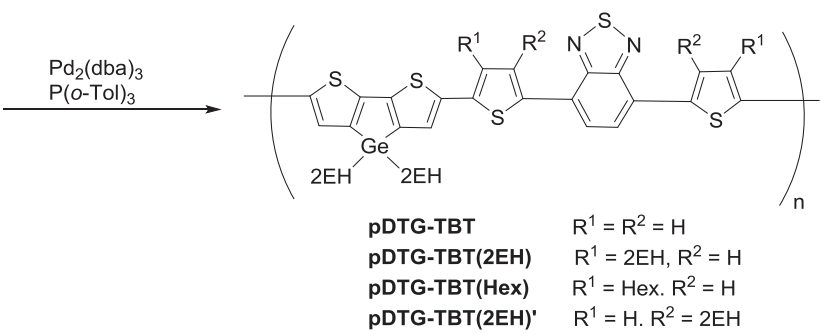

Scheme 2 Polymer synthesis.

show how the molecular weight and the substitution affect the photovoltaic properties.

\section{RESULTS AND DISCUSSION}

Polymer synthesis

Polymers with alternating DTG and alkyl-substituted TBT units were synthesized as black solids by a Stille coupling in refluxing chlorobenzene using $\mathrm{Pd}_{2}(\mathrm{dba})_{3} /(o-\mathrm{Tol})_{3} \mathrm{P}$ as the catalyst, similarly to what was previously reported for pDTG-TBT (Scheme 2, see Supplementary Information for details). ${ }^{19}$ Table 1 summarizes the properties of the polymers. They showed good solubility in chloroand $o$-dichlorobenzene and were moderately soluble in chloroform and tetrahydrofuran but insoluble in toluene and ethanol. As expected, GPC of the polymers showed high molecular weights of $M_{\mathrm{n}}=26000-57000$ with an approximate polydispersity index $\left(M_{\mathrm{w}} / M_{\mathrm{n}}\right)$ of 2 , relative to polystyrene standards.

We also investigated the preparation of pDTG-TBT employing DTGSn monomer that was purified by preparative GPC and showed no impurity signals in the nuclear magnetic resonance spectra. The reaction mixture was filtered and the soluble organic products were purified by precipitation combined with Soxhlet fractional extraction. GPC analysis of the polymer prepared by this method (pDTG-TBT1) revealed an approximately two times higher molecular weight $\left(M_{\mathrm{n}}\right)$ and a much smaller polydispersity $\left(M_{\mathrm{w}} / M_{\mathrm{n}}\right)$ compared with the previously prepared polymer (pDTG-TBT0; Table 1), ${ }^{19}$ although no

\begin{tabular}{|c|c|c|c|c|c|c|}
\hline \multirow[b]{2}{*}{ Polymer } & \multirow{2}{*}{$\begin{array}{l}\text { Yield } \\
(\%)^{a}\end{array}$} & \multirow[b]{2}{*}{$\mathrm{M}_{n}^{\mathrm{b}}\left(\mathrm{M}_{w} \mathrm{M}_{n}\right)$} & \multicolumn{2}{|c|}{$U V$-vis $\lambda_{\max }(\mathrm{nm})$} & \multirow[b]{2}{*}{$\mathrm{T}_{d}{ }^{5}\left({ }^{\circ}\right)^{\mathrm{c}}$} & \multirow{2}{*}{$\begin{array}{c}\text { HOMO, } \\
\text { LUMO }(\mathrm{eV})^{\mathrm{d}}\end{array}$} \\
\hline & & & in $\mathrm{CHCl}_{3}$ & Film & & \\
\hline pDTG-TBT1 $1^{\mathrm{e}}$ & 52 & $7100(1.1)$ & 625 & 664 & 412 & $-5,-3.5$ \\
\hline pDTG-TBTO ${ }^{f}$ & 50 & $3800(3.2)$ & 585 & 620 & 382 & $-5.1,-3.5$ \\
\hline pDTG-TBT(2EH) & 91 & $26000(2)$ & 583 & 626 & 407 & $-5.2,-3.6$ \\
\hline pDTG-TBT(Hex) & 95 & 27000 (2) & 591 & 662 & 418 & $-5,-3.4$ \\
\hline pDTG-TBT(2EH)' & 60 & 57000 (2) & 516 & 532 & 400 & $-5.3,-3.3$ \\
\hline
\end{tabular}

Abbreviations: DTG-TBT, dithienogermole-dithienylbenzothiadiazole; HOMO, highest occupied molecular orbital; GPC, gel permeation chromatography; LOMO, lowest unoccupied molecular orbital.

${ }^{a}$ After reprecipitation.

Determined by GPC, relative to polystyrene standards.

$5 \%$ Weight less temperature.

Estimated from CV onset potential on polymer film containing tetrabutyl perchlorate in acetonitrile and optical band gap.

ePresent work.

fLiterature data (Hwang et al. ${ }^{19}$ ).

evident differences were observed between the nuclear magnetic resonance spectra of pDTG-TBT1 and pDTG-TBT0. Polymer pDTG-TBT1 was obtained as a black solid that were soluble in chlorobenzene and dichlorobenzene, moderately soluble in chloroform and slightly soluble in tetrahydrofolate, but insoluble in toluene, ethanol and acetone.

UV-vis absorption spectra of the polymers were measured in chloroform and as thin spin-coated films, revealing broad absorption bands. Interestingly, pDTG-TBT1 showed a largely red-shifted absorption band in comparison with pDTG-TBT0, presumably because of the higher molecular weight. As presented in Table 1 and Figure 1, the introduction of alkyl groups on the TBT units in pDTG-TBT(2EH) and pDTG-TBT(Hex) resulted in blue shifts of the UV-vis absorption maxima compared with pDTG-TBT1. It is likely that the alkyl substituents on the TBT thiophene rings in these polymers hinder the backbone planarity by steric interaction with DTG C-H bonds at the $\beta$-positions. The position of the alkyl groups also affected the UV-vis spectral profile. pDTS-TBT(2EH)' showed an even more blue-shifted absorption band, suggesting significant steric repulsion between the 2-ethylhexyl and BT units that decreased the polymer backbone planarity. A similar dependence of UV-vis absorption properties on the position of substituents was reported for triphenylamine-TBT alternating polymers. ${ }^{6}$

Measuring the absorption spectra of the polymers as films moved the absorption bands to lower energies, likely because of interchain 
interactions in the polymers in the solid state leading to increased planarity, and/or through space electronic $\pi-\pi$ interactions. The largest state-dependent (that is, solution or film) energy shift of $71 \mathrm{~nm}$ was observed for pDTG-TBT(Hex). The absorption maximum of this polymer film was at nearly the same energy as that of pDTGTBT1. Interestingly, the state-dependent red shift for pDTG-TBT1 $(39 \mathrm{~nm})$ was larger than that of pDTG-TBT0 $(35 \mathrm{~nm})$, indicating stronger interchain interactions in the solid state for this polymer, presumably reflecting its higher molecular weight. We also measured cyclic voltammograms of the polymer films containing tetrabutylammonium perchlorate, and the HOMO and LUMO energy levels were estimated on the basis of CV (cyclic voltammogram) anodic peak and UV-vis absorption onsets, which are listed in Table 1. The HOMO levels are slightly higher than or comparable to that of PEDOT:PSS $(-5 \mathrm{eV})$, whereas the LUMOs lie at a higher level than that of $\mathrm{PC}_{71} \mathrm{BM}$ (from -3.7 to $-4 \mathrm{eV}$ ), suggesting potential applicability as the host polymers for BHJ-PSCs (vide infra).

The thermal stability of the polymers was investigated by thermogravimetric analysis (TGA) in nitrogen. Five percent weight loss temperatures $\left(T_{\mathrm{d}}^{5}\right)$ were noted and are listed in Table 1. No unambiguous signals were observed by differential scanning calorimetry (DSC). TGA of pDTG-TBT1 revealed $T_{\mathrm{d}}{ }^{5}=412^{\circ} \mathrm{C}$, which was higher than that of pDTG-TBT0 by $30^{\circ} \mathrm{C}$. The other polymers with alkylated TBT units also exhibited good thermal stability and had $T_{\mathrm{d}}{ }^{5}$ values higher than $400{ }^{\circ} \mathrm{C}$, most likely because of their high molecular weights.

\section{Photovoltaic properties}

BHJ-type PSCs using the present polymers as the host materials and $\mathrm{PC}_{71} \mathrm{BM}$ as the guests were fabricated with the structure of ITO (indium

\section{pDTG-TBT(2EH)' pDTG-TBT(2EH)}
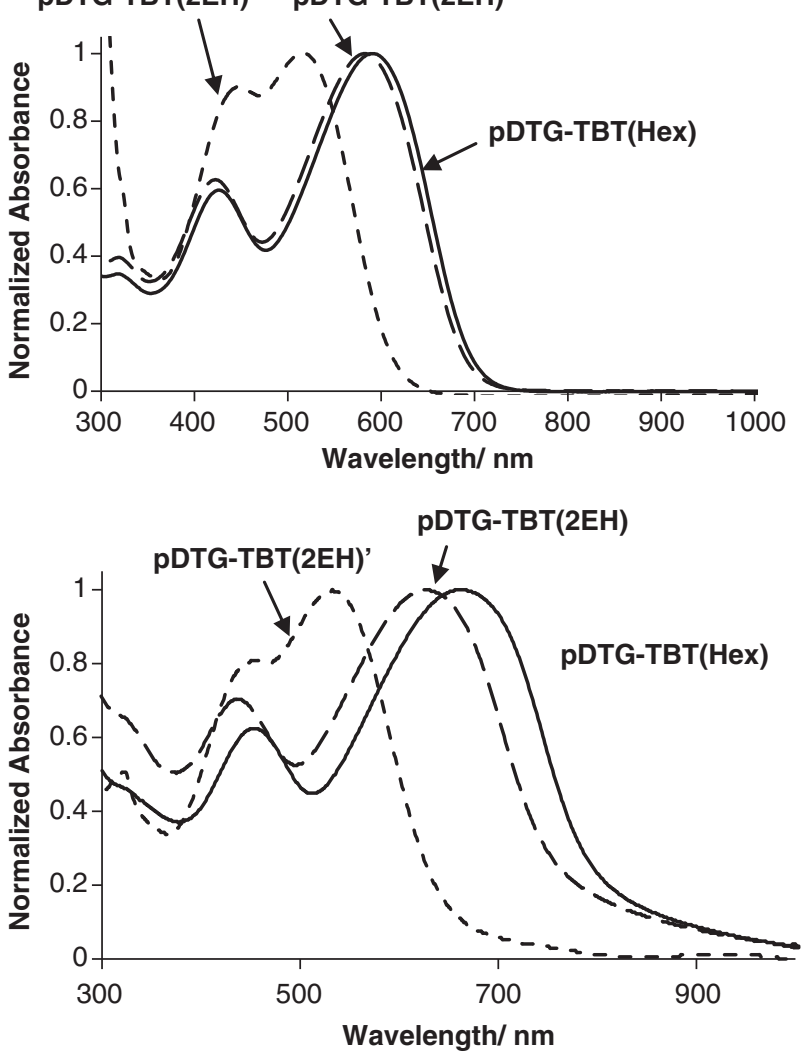

Figure 1 UV-vis absorption spectra of DTG-TBT polymers in chloroform (top) and as films (bottom). tin oxide, $150 \mathrm{~nm}$ )/PEDOT:PSS (poly(3,4-ethylenedioxythiophene):poly(styrene sulfonate), $30 \mathrm{~nm}) / \mathrm{DTG}-\mathrm{TBT}$ polymer:PC $\mathrm{P}_{71} \mathrm{BM}($ c.a. $80 \mathrm{~nm}) /$ $\operatorname{LiF}(0.5 \mathrm{~nm}) / \mathrm{Al}(80 \mathrm{~nm})$ and an active area of $0.25 \mathrm{~cm}^{2}$, in a manner similar to that previously reported. ${ }^{19}$ As expected, the cells showed clear photovoltaic properties. The fabrication conditions were optimized by screening some of the parameters (that is, polymer: $\mathrm{PC}_{71} \mathrm{BM}$ ratio, spincoating solvent and thickness of the active layer). Annealing the active layers as well as the devices did not affect the results. The photovoltaic parameters of the cells that were fabricated under the conditions optimized for each of the cells are listed in Table 2. Notably, the cell with pDTG-TBT1 exhibited better performance than the one with pDTG-TBT0 $\quad\left(J_{\mathrm{sc}}=5.47 \mathrm{~mA} \mathrm{~cm}^{-2}, \quad V_{\mathrm{oc}}=0.59 \mathrm{~V}, \quad \mathrm{FF}=0.48\right.$, $\mathrm{PCE}=1.55 \%),{ }^{19}$ likely because of its higher structural regularity, higher molecular weight and/or lower polydispersity. It seems likely that structural regularity minimizes the carrier-trapping sites. High molecular weight and low polydispersity would also exert an influence on the film morphology to enhance the photocurrent.

Table 2 Cell parameters of BHJ-PSCs with DTG-TBT polymers ${ }^{\mathrm{a}}$

\begin{tabular}{|c|c|c|c|c|c|c|c|}
\hline Polymer & $\begin{array}{l}\text { Polymer: } \\
P C_{71} B M^{\mathrm{b}}\end{array}$ & Solv & $\begin{array}{l}\text { Thk } \\
(n m)\end{array}$ & $\begin{array}{c}\mathrm{J}_{S c} \\
\left(m A c m^{-2}\right)\end{array}$ & $\begin{array}{l}\mathrm{V}_{o c} \\
(V)\end{array}$ & $F F$ & $\begin{array}{l}P C E \\
(\%)\end{array}$ \\
\hline pDTG-TBT1 & $1: 2$ & CB & 68 & 13.46 & 0.52 & 0.55 & 3.83 \\
\hline pDTG-TBT(2EH) & $1: 2$ & DCB & 82 & 3.29 & 0.79 & 0.43 & 1.16 \\
\hline pDTG-TBT(Hex) & $1: 2$ & DCB & 88 & 10.12 & 0.54 & 0.66 & 3.58 \\
\hline $\begin{array}{l}\text { pDTG- } \\
\text { TBT(2EH })^{\prime}\end{array}$ & $1: 3.6$ & DCB & 67 & 5.56 & 0.58 & 0.32 & 1.04 \\
\hline
\end{tabular}

Abbreviations: BHJ-PC, bulk heterojunction-type polymer solar cell; $\mathrm{CB}$, chlorobenzene; DCB, o-dichlorobenzene; 2.5 vol\% of diiodooctane was employed as a processing additive; DTG-TBT, dithienogermole-dithienylbenzothiadiazole.

aWith the cell structure of ITO $(150 \mathrm{~nm}) / \mathrm{PEDOT}$ :PSS $(30 \mathrm{~nm}) / \mathrm{DTG}-\mathrm{BTA}$ polymer: $\mathrm{PC}{ }_{71} \mathrm{BM}$ $($ ca. $80 \mathrm{~nm}) / \mathrm{LiF}(0.5 \mathrm{~nm}) / \mathrm{Al}(80 \mathrm{~nm})$ with an active area of $0.25 \mathrm{~cm}^{2}$.

bwt ratio.
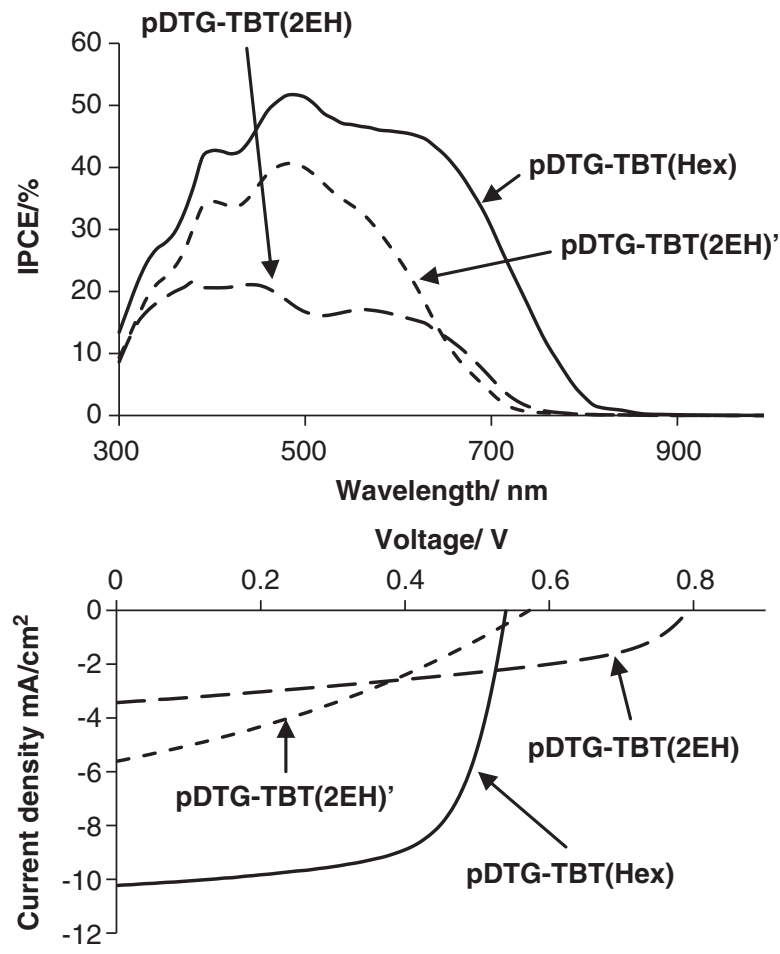

Figure 2 IPCE spectra (top) and $J-V$ curves (bottom) of alternating polymers with DTG and alkyl-substituted TBT units. 
For the cells based on the polymers with alkyl-substituted TBT, the PSC with pDTG-TBT(Hex) provided the highest PCE, arising primarily from a relatively high photocurrent. However, the cell with pDTG-TBT1 exhibited even higher photocurrent (vide supra), leading to a higher PCE by $0.25 \%$, although the voltage was slightly decreased. Alkyl groups on TBT units may hinder the interchain carrier hopping, which would suppress the photocurrent. Incident photon-to-current conversion efficiency (IPCE) spectra and current density-voltage ( $J$ $V)$ curves of the present cells are shown in Figure 2. The device with pDTG-TBT(2EH) gave a higher $V_{\text {oc }}$ than that with pDTG-TBT(Hex), presumably because of the twisted structure of pDTG-TBT(2EH), which lowered the HOMO energy level (Table 1). However, twisting also led to its blue-shifted absorption band and rather weak $\pi$ conjugation in the polymer, which decreased the photocurrent. The cell with pDTG-TBT(2EH)' showed moderate $J_{\mathrm{sc}}$ and $V_{\mathrm{oc}}$, thus giving a PCE similar to that of the pDTG-TBT(2EH)-based one. The reason for the lower $V_{\mathrm{oc}}$ with pDTG-TBT(2EH)', despite having a low-lying HOMO because of its twisted structure (Table 1), is still unclear. However, other factors such as the polymer interface and morphology could also be responsible for the low fill factor.

\section{CONCLUSIONS}

We successfully prepared high molecular weight DTG-TBT-alternating polymers with alkyl substituents on the TBT units, which showed good solubility in organic solvents with appropriate polarity. Interestingly, the polymer electronic states were significantly affected by the nature and positions of the substituents, and the conjugation was suppressed in the following order: pDTG-TBT(Hex)>pDTGTBT (2EH) > pDTG-TBT(2EH)'. Of those polymers, pDTGTBT(Hex) showed markedly enhanced $\pi$ conjugation as films compared with that in the solution phase. Notably, pDTG-TBT(Hex) exhibited photovoltaic properties that were similar to those of pDTGTBT with non-substituted TBT units, despite the fact that alkyl substitution may suppress the carrier mobility by disturbing the interchain carrier hopping. In D-A type $\pi$-conjugated polymers, TBT is often employed as an acceptor fragment. ${ }^{1,6,19,22-25}$ However, the introduction of substitution-free TBT usually leads to solubility problems. Our results showing that the introduction of $n$-alkyl groups on the $3,3^{\prime}$-positions of the thiophene rings increases the solubility of the polymer without significantly decreasing its photovoltaic properties seem to be an important finding that could be applied to other $\pi$-conjugated materials with TBT units.

\section{CONFLICT OF INTEREST}

The authors declare no conflict of interest.

\section{ACKNOWLEDGEMENTS}

This work was supported by the Adaptable and Seamless Technology Transfer Program through Target-driven R\&D, JST and a Grant-in-Aid for Scientific Research (B) (No. 23350097) from the Ministry of Education, Culture, Sports, Science and Technology, Japan. F-BZ thanks his home affiliation, the Key Laboratory of Organosilicon Chemistry and Material Technology of the Ministry of Education, Hangzhou Normal University, Hangzhou 310012, China for the permission to join Hiroshima University as a guest researcher.

1 Li, Y.-F. Molecular design of photovoltaic materials for polymer solar cells: toward suitable electronic energy levels and broad absorption. Acc. Chem. Res. 45, 723 (2012).
2 Schilinsky, P., Asawapiron, U., Scherf, U., Biele, M. \& Brabee, C. J. Influence of the molecular weight of poly(3-hexylthiophene) on the performance of bulk heterojunction solar cells. Chem. Mater. 17, 2175 (2005).

$3 \mathrm{Ma}$, W., Kim, J. Y., Lee, K. \& Heeger, A. Effect of the molecular weight of poly(3hexylthiophene) on the morphology and performance of polymer bulk heterojunction solar cells. Macromol. Rapid Commun. 28, 1776 (2007).

4 Hiorns, R. H., Bettignies, R. D., Leroy, J., Bailly, S., Fieron, M., Sentein, C., Khoukh, A., Preud'homme, H. \& Dagron-Lartigau, C. High molecular weights, polydispersities, and annealing temperatures in the optimization of bulk-heterojunction photovoltaic cells based on poly(3-hexylthiophene) or poly(3-butylthiophene). Adv. Funct. Mater. 16, 2263 (2006).

5 Tinti, F., Sabir, K., Gazzano, M., Righi, S., Ulbricht, C., Usluer, Ö., Pokorna, V., Cimrove, V., Yohannes, T., Egbe, D. A. M. \& Camaioni, N. Tuning the properties of an anthracene-based PPE-PPV copolymer by fine variation of its macromolecular parameters. RSC Adv. 3, 6972 (2013)

6 Yasuda, T., Shinohara, Y., Matsuda, T., Han, L. \& Ishi-i, T. Synthesis and photovoltaic properties of amorphous polymers based on dithienylbenzothiadiazole-triphenylamine with hexyl side chains on different positions of thienyl groups J. Polym. Sci. A 51, 2536 (2013).

7 Hou, J., Chen, H.-Y., Zhang, S., Li, G. \& Yang, Y. Synthesis, characterization, and photovoltaic properties of a low band gap polymer based on silole-containing polythiophenes and 2,1,3-Benzothiadiazole. J. Am. Chem. Soc. 130, 16144 (2008).

8 Ohshita, J. Conjugated oligomers and polymers containing dithienosilole units. Macromol. Chem. Phys. 210, 1360 (2009).

9 Huo, L., Chen, H.-Y., Hou, J., Chen, T. L. \& Yang, Y. Low band gap dithieno[3,2-b:2', 3' d]silole-containing polymers, synthesis, characterization and photovoltaic application. Chem. Commun. 5570 (2009).

10 Zhang, M., Guo, X., Zhang, Z.-G. \& Li, Y. D-A copolymers based on dithienosilole and phthalimide for photovoltaic materials. Polymer (Guildf) 52, 5464 (2011).

11 Peet, J., Wen, L., Byrne, P., Rodman, S., Forberich, K., Shao, Y., Drolet, N. \& Gaudiana, R. Bulk heterojunction solar cells with thick active layers and high fill factors enabled by a bithiophene-co-thiazolothiazole push-pull copolymer. Appl. Phys. Lett. 98, 043301 (2011)

12 Subramaniyan, S., Xin, H., Kim, F. S., Shoaee, S., Durrant, J. R. \& Jenekhe, S. A. Effects of side chains on thiazolothiazole-based copolymer semiconductors for high performance solar cells. Adv. Energy Mater. 1, 854 (2011).

13 Subramaniyan, S., Xin, H., Kim, F. S. \& Jenekhe, S. A. New thiazolothiazole copolymer semiconductors for highly efficient solar cells. Macromolecules 44, 6245 (2011).

14 Chu, T.-Y., Lu, J., Beaupre, S., Zhang, Y., Pouliot, J.-R., Wakim, S., Zhou, J., Leclerc, M., Li, Z., Ding, J. \& Tao, Y. Bulk heterojunction solar cells using Thieno[3,4-c]pyrrole4,6-dione and Dithieno[3,2-b:2' $3^{\prime}$-d]silole copolymer with a power conversion efficiency of 7.3\%. J. Am. Chem. Soc. 133, 4250 (2011).

15 le, Y., Huang, J., Uetani, Y., Karakawa, M. \& Aso, Y. Synthesis, properties, and photovoltaic performances of donor-acceptor copolymers having dioxocycloalkeneannelated thiophenes as acceptor monomer units. Macromolecules 45, 4564 (2012).

16 Guo, X., Zhou, N., Lou, S. J., Hennek, J. W., Ortiz, R. P., Butler, M. R., Boudreault, P.L. T., Strzalka, J., Morin, P.-O., Leclerc, M., Navarrete, J. T. L., Ratner, M. A., Chen, L. $X$, Chang, P. H. Facchetti, A. \& Marks, T. J. Bithiopheneimide-dithienosilole/ dithienogermole copolymers for efficient solar cells: information from structureproperty-device performance correlations and comparison to Thieno[3,4-c]pyrrole4,6-dione analogues. J. Am. Chem. Soc. 134, 18427 (2012).

17 Ohshita, J., Hwang, Y.-M., Mizumo, T., Yoshida, H., Ooyama, Y., Harima, Y. \& Kunugi, $\mathrm{Y}$. Synthesis of dithienogermole-containing $\pi$-conjugated polymers and applications to photovoltaic cells. Organometallics 30, 3233 (2011).

18 Ohshita, J., Miyazaki, M., Zhang, F.-B., Tanaka, D. \& Morihara, Y. Synthesis and properties of dithienometallole-pyridinochalcogenadiazole alternate polymers. Polymer J. 45, 979 (2013)

19 Hwang, Y.-M , Ohshita, J., Harima, Y Mizumo, T, Ooyama, Y., Morihara, Y., Izawa, $T$., Sugioka, T. \& Fujita, A. Synthesis, characterization, and photovoltaic applications of dithienogermole-dithienylbenzothiadiazole and -dithienylthiazolothiazole copolymers. Polymer (Guildf) 52, 3912 (2011).

20 Amb, C. M., Chen, S., Graham, K. R., Subbiah, J., Small, C. E., So, F. \& Reynolds, J. R. Dithienogermole as a fused electron donor in bulk heterojunction solar cells. J. Am. Chem. Soc. 133, 10062 (2011).

21 Small, C. E., Chen, S., Subbiah, J., Amb, C. M., Tsang, S. W., Lai, T. H., Reynolds, J. R. \& So, F. High-efficiency inverted dithienogermole-thienopyrrolodione-based polymer solar cells. Nat. Photon. 6, 115 (2012).

22 Hou, Q., Xu, Y., Yang, M., Peng, J. B. \& Cao, Y. Novel red-emitting fluorene-based copolymers. J. Mater. Chem. 12, 2887 (2002).

23 Zhou, J., Xie, S., Amond, E. F. \& Becker, M. L. Tuning energy levels of low bandgap semi-random two acceptor copolymers. Macromolecules 46, 3391 (2013).

24 Qu, B., Yang, H., Tian, D., Liu, H., Cong, Z., Gao, C. Chen, Z., Xiao, L., Gao, Z., Wei, W. \& Gong, Q. Synthesis of two benzo[1,2-b:3,4-b']dithiophene-based conjugated polymers with different side chains and their applications in photovoltaic devices. Synth. Met. 162, 2020 (2012).

25 Xiao, Y., Lu, X., Tan, L.-W., Ong, K. S. \& He, C. Thermally stable red electroluminescent hybrid polymers derived from functionalized silsesquioxane and 4,7-bis(3-ethylhexyl2-thienyl)-2,1,3-benzothiadiazole. J. Polym. Sci. A 47, 5661 (2009). 